\title{
Tissue effects in vessel sealing and transection from an ultrasonic device with more intelligent control of energy delivery
}

This article was published in the following Dove Press journal:

Medical Devices: Evidence and Research

13 September 2013

Number of times this article has been viewed

\author{
Duan Broughton' \\ Alissa L Welling' \\ Emily H Monroe' \\ Kristen Pirozzi ${ }^{2}$ \\ John B Schulte' \\ Jeffrey W Clymer' \\ 'Ethicon Endo-Surgery, Inc., Cincinnati, \\ $\mathrm{OH},{ }^{2}$ University of Central Florida, \\ Orlando, FL, USA
}

Background: Ultrasonic surgical devices have been demonstrated to provide excellent hemostasis, efficient transection, minimal lateral thermal damage, low smoke generation, and no risk of electrical current passage to the patient. These benefits originate from the inherent characteristics of the ultrasonic mechanism, but further improvements were thought possible through optimization of the energy delivery during application. The study reported here compared a new ultrasonic device, Harmonic $\mathrm{ACE}^{\circledR}+$ Shears with Adaptive Tissue Technology, with a commercial predicate device, Harmonic ACE ${ }^{\circledR}$ Shears (both Ethicon Endo-Surgery, Inc., Cincinnati, OH, USA).

Methods: Devices were evaluated in an in vivo porcine model intraoperatively and after a 30 -day survival period. Both devices were used to seal a variety of vessels $1-5 \mathrm{~mm}$ in diameter, and compared for hemostasis, histological thermal damage, and adhesion formation. Sealed vessels were evaluated ex vivo for burst pressure, and visual obstruction caused by plumage from device application was assessed quantitatively.

Results: ACE+ produced significantly less thermal damage, had fewer adhesions, offered faster transection, involved less visual obstruction, and had higher burst pressures than the predicate device. All vessel seals, evaluated over the course of a 30-day survival period in pigs, remained intact.

Conclusion: The new Adaptive Tissue Technology algorithm assists the surgeon in achieving better control of energy delivery to the tissue, sealing vessels with supra-physiological burst pressures, and low thermal damage. These preclinical results with Harmonic ACE+ may translate into meaningful clinical benefits, providing greater precision along with more efficient and effective cutting and coagulation in open or laparoscopic procedures.

Keywords: ultrasonic, Harmonic, ACE, vessel sealing, burst pressure, adhesions, Adaptive Tissue Technology

\section{Introduction}

Since its inception, vessel sealing with ultrasonic devices has been recognized as having many advantages over conventional electrosurgery. ${ }^{1}$ The purely mechanical action of the ultrasonic end effector eliminates the passage of any current to or through the patient, reducing the risk of nerve damage. ${ }^{2}$ Hemostasis is achieved with minimal lateral thermal damage, ${ }^{3}$ and minimal smoke (mist or vapor) is produced. ${ }^{4}$

Critical to the formation of a strong and durable vessel seal is the energy profile delivered by an ultrasonic device. Building on the foundation of basic Harmonic ${ }^{\circledR}$ functionality, Adaptive Tissue Technology has been developed to provide greater precision through improved energy delivery. ${ }^{5}$ Exclusive to Harmonic devices, Adaptive Tissue Technology actively monitors the instrument during use and enables the system
Correspondence: Jeffrey W Clymer Ethicon Endo-Surgery, Inc., 4545 Creek Rd, Cincinnati, $\mathrm{OH} 45242$, USA

$\mathrm{Tel}+\mathrm{I} 5133373318$

Email jclymer@its.jnj.com 
to respond intelligently to varying tissue conditions. With the Harmonic $\mathrm{ACE}^{\circledR}+$ Shears (Ethicon Endo-Surgery, Inc., Cincinnati, OH, USA; Figure 1), Adaptive Tissue Technology delivers greater precision by regulating energy delivery when needed. During a transection and as tissue divides, the blade contacts the tissue pad of the device and the blade temperature typically begins to increase more rapidly. Adaptive Tissue Technology responds by reducing the power output and providing enhanced feedback with a change in an audible activation tone. Hemostasis is achieved and unnecessary power output that could potentially lead to thermal injury is reduced. Additionally, the Harmonic ACE+ Shears feature a tapered, coated blade that brings precision to multiple surgical jobs, including dissection, sealing, transection, and grasping.

This study was undertaken to compare the Harmonic ACE+ Shears with Adaptive Tissue Technology, with the previous version of the Harmonic $A_{C E}{ }^{\circledR}$ Shears (Ethicon Endo-Surgery, Inc.,). It was hypothesized that the technological advances incorporated into the new device would maintain rates of initial hemostasis and seal durability, while decreasing transection time, thermal damage, smoke formation, and the risk of adhesion formation during wound healing.

\section{Methods}

All procedures were reviewed and the animals approved for use in the study by the Ethicon Endo-Surgery Institutional Animal Care and Use Committee. A porcine model was chosen based on the similarity of its blood vessel composition and sealing to that of humans.

The primary comparison was between Harmonic ACE Shears of lengths 23 and $36 \mathrm{~cm}$, and the new Harmonic ACE+ Shears with Adaptive Tissue Technology of the same

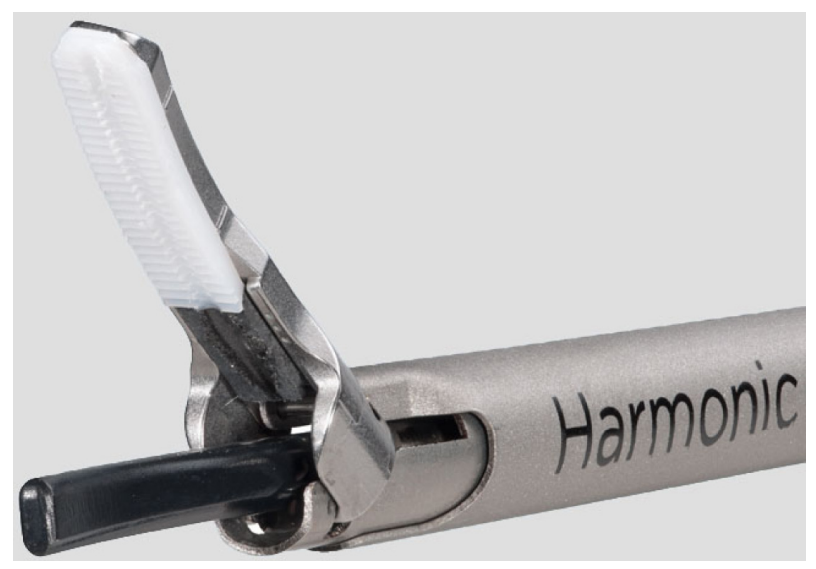

Figure I End effector of the Harmonic $\mathrm{ACE}^{\oplus}+$ Shears (Ethicon Endo-Surgery, Inc., Cincinnati, OH, USA). lengths. For the acute procedures, both the 23 and $36 \mathrm{~cm}$ lengths of the new and predicate devices were utilized and compared; for the survival study, the $36 \mathrm{~cm}$ lengths of each were utilized and compared.

Vessel sealing was tested for ACE versus ACE+ in porcine carotid, gastroepiploic, splenic, and short gastric arteries, veins and pedicles, at generator power level settings of 3 or 5 . Intraoperative measurements included vessel size, transection time, initial hemostasis, and tissue sticking. Postoperatively, thermal damage was assessed via hematoxylin and eosin (H\&E) staining. A subset of the animals was allowed to survive up to 30 days, at which time hemostasis was checked at each vessel seal after raising the blood pressure to $>200 \mathrm{mmHg}$ to induce a simulated hypertensive crisis. At necropsy, a visual examination for adhesions was also performed.

Burst pressures were measured on porcine carotid vessels of diameter 3-5 mm sealed with ACE $(n=80)$ or ACE+ $(\mathrm{n}=80)$ at power level 3, via injection of saline at a rate of $26 \mathrm{~mL} / \mathrm{min}$ using a proprietary system.

Visual obstruction from smoke or haze was measured by a conventional method. ${ }^{6}$ In a specially constructed chamber, the devices were activated for 3 seconds on the maximum power setting, which was considered a worst-case scenario. Five devices each of ACE and ACE+ were used, and 12 runs were performed per device. The curved version of each device was used, as this represents the worst-case scenario for plume generation, since curved blades produce turbulent plume flow, whereas straight blades produce laminar flow. Obstruction was recorded using a Tele Pack X system with a Telecam camera (Karl Storz GmbH and Co, KG, Tuttlingen, Germany). The recording was converted with Movavi ${ }^{\circledR}$ Video Converter software, version 12 (Movavi, Saint Louis, MO, USA) and analyzed with ImageJ image processing and analysis software, version 1.48b (National Institutes of Health, Bethesda, MD, USA) to measure the amount of pixel obstruction observed in real-time.

Continuous variables, such as transection time or thermal damage, were compared using a $t$-test or analysis of covariance (ANCOVA), as appropriate, and binomial variables, such as hemostasis and adhesions, were compared using Fisher's exact test.

\section{Results}

There were no complications during the acute procedures and all but one animal in the 30-day study survived until the appointed time. A single animal was found deceased after 18 days due to unrelated causes; all vessel seals were healing normally with no evidence of bleeding. 
The distribution of vessel types used was not significantly different between ACE and ACE+. The diameters of vessels ranged from 1 to $5 \mathrm{~mm}$, and the mean vessel diameter was $3.4 \mathrm{~mm}$ for both devices. There was no difference in the distribution of generator power settings used between the two devices.

A tabulation of key results is given in Table 1. Initial hemostasis was high for both devices $(97.7 \%$ for ACE and $99.0 \%$ for ACE + ) and not significantly different. The new Harmonic ACE+ Shears were $21 \%$ faster $(P<0.001)$ in transection time than the predicate shears. Neither device exhibited any tissue sticking. As measured by H\&E staining, the mean zone of adventitial collagen denaturation (thermal damage) for ACE + was $<2 \mathrm{~mm}$, and $23 \%$ less than ACE $(P<0.001$; Figure 2).

At the end of the survival period, both devices showed $100 \%$ hemostasis of all seals after a simulated hypertensive crisis $>222 \mathrm{mmHg}$. The predicate device, ACE, had adhesion formation at $39.4 \%$ of vessel seals, while the new ACE+ had no adhesion formation at any site $(P<0.001)$.

Burst pressures from ex vivo testing were significantly higher for ACE + than for ACE $(P=0.002)$. Visual obstruction from smoke/mist was reduced by $40.5 \%$ for ACE+ compared with ACE $(P<0.001)$.

\section{Discussion}

In the preclinical study reported here, we examined the effects of incorporation of Adaptive Tissue Technology into the Harmonic platform. This technology provides the system with the ability to monitor the thermal condition of the blade and to identify conditions correlative to unnecessary thermal energy - namely, rapid increases in heat flux into the blade from action directly against the tissue pad rather than against tissue. When minimal tissue remains in the jaw, the generator decreases its power output and an audible change in tone provides enhanced feedback to the user. This more intelligent

Table I ACE ${ }^{\circledR}$ versus $\mathrm{ACE}^{\circledR}+$ results summary

\begin{tabular}{llll}
\hline Measure & ACE & ACE + & P-value \\
\hline Transection time* & $5.7 \mathrm{~s}$ & $4.5 \mathrm{~s}$ & $<0.00 \mathrm{I}$ \\
Initial hemostasis & $127 / 130$ & $95 / 96$ & 0.639 \\
Thermal damage** & $2.19 \mathrm{~mm}$ & $1.69 \mathrm{~mm}$ & $<0.001$ \\
Survival hemostasis & $33 / 33$ & $32 / 32$ & 1.000 \\
Adhesions & $13 / 33$ & $0 / 32$ & $<0.001$ \\
Burst pressure & $1,208 \mathrm{mmHg}$ & $1,514 \mathrm{mmHg}$ & 0.002 \\
Average visual & $4.57 \%$ & $2.72 \%$ & $<0.001$ \\
obstruction & & & \\
\hline
\end{tabular}

Notes: *Adjusted for vessel size and device length via analysis of covariance (ANCOVA); **adjusted for device length and vessel type via ANCOVA.

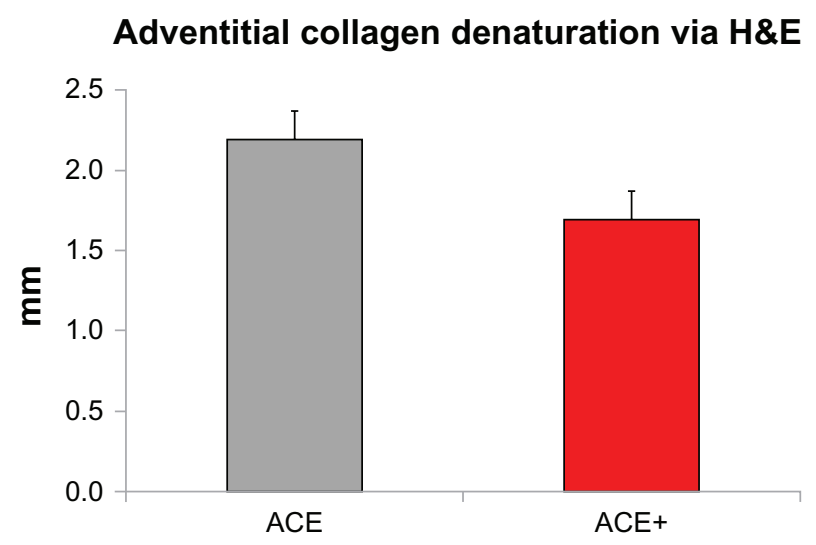

Figure 2 Mean thermal damage via hematoxylin and eosin (H\&E) for $A C E^{\circledR}$ and $\mathrm{ACE}^{\circledR}+; \mathrm{ACE}+$ had a significantly narrower band of thermal damage than ACE. Note: Error bars represent the $95 \%$ confidence interval.

technology reduces unnecessary power application that could potentially lead to excess thermal injury.

Historically, Harmonic ACE Shears have been shown to provide excellent hemostasis, ${ }^{7}$ and strong, durable seals. ${ }^{8}$ In the current study, we observed that the Harmonic ACE+ Shears maintain these properties, with $99 \%$ success in initial hemostasis in vessels up to $5 \mathrm{~mm}$ diameter, and $100 \%$ durability of vessel seals up to 30 days postoperatively. Burst pressures for $\mathrm{ACE}+$ were $25 \%$ higher than for $\mathrm{ACE}$, although blade temperatures were significantly lower.

In contrast with electrosurgical methods, ultrasonic devices are recognized as producing less tissue sticking during use. ${ }^{9}$ The improvements in ACE+ were shown in this study to maintain this useful characteristic, critical to operational speed and efficiency, with no observed tissue sticking. ACE + produced less smoke and visual obstruction, which can also lead to increased operation efficiency.

The Harmonic ACE+ Shears transected tissue 21\% faster than the previous model. This significant difference is in part due to thermal monitoring and enhanced audible feedback to the user. Based on the lower temperatures produced by ultrasonic devices, it has been shown that they produce less thermal damage than electrosurgical devices, ${ }^{10}$ and that the reduced thermal damage has resulted in less inflammation ${ }^{11}$ and faster wound healing. ${ }^{12}$ With ACE+, the improved energy management has further reduced the thermal damage, leading to a $23 \%$ decrease and a mean zone of collagen denaturation $<2 \mathrm{~mm}$.

The inflammatory reaction due to collagen denaturation has been implicated in postoperative adhesion formation. ${ }^{13}$ The decreased thermal damage caused by ACE+ may be responsible for the reduced rate of adhesion formation compared with that of the previous version of the device. 
It is also possible that the coated blade aids in the prevention of adhesions. In any case, no adhesions were observed after a 30-day survival period with $\mathrm{ACE}+$ in this preclinical model.

\section{Conclusion}

The new Harmonic ACE+ Shears continue to provide excellent vessel sealing hemostasis and durability, with no tissue sticking. Incorporation of Adaptive Tissue Technology has optimized the delivery of energy to provide enhanced precision, resulting in reduced tissue damage, faster dissection, better procedure visualization, and lowered risk of postoperative adhesion formation.

\section{Disclosure}

Duan Broughton, Alissa L Welling, Emily H Monroe, Kristen Pirozzi, John B Schulte, and Jeffrey W Clymer were employed during the execution of this study by Ethicon Endo-Surgery, Inc., the manufacturer of the Harmonic ACE and ACE+ Shears. The authors report no other conflicts of interest in this work.

\section{References}

1. Amaral JF. Laparoscopic application of an ultrasonically activated scalpel, Issue no. 2. Gastrointest Endosc Clin North Am. 1993;3:381-391.

2. Chen C, Kallakuri S, Vedpathak A, et al. The effects of ultrasonic and electrosurgery devices on nerve physiology. Br J Neurosurg. 2012;26(6): 856-863.
3. Phillips CK, Hruby GW, Durak E, et al. Tissue response to surgical energy devices. Urology. 2008;71(4):744-748.

4. Schmidbauer S, Hallfeldt KK, Sitzmann G, Kantelhardt T, Trupka A. Experience with ultrasound scissors and blades (UltraCision) in open and laparoscopic liver resection. Ann Surg. 2002;235(1):27-30.

5. Raghavan D, Howington JA, Broughton D, Henderson CE, Clymer JW. Comparison of two ultrasonic coagulating shears in sealing pulmonary vessels. Open Access Surg. 2013;6:15-21.

6. Kim FJ, Sehrt D, Pompeo A, Molina WR. Comparison of surgical plume among laparoscopic ultrasonic dissectors using a real-time digital quantitative technology. Surg Endosc. 2012;26(12):3408-3412.

7. Yamada S, Yoshino K, Inoue H. New-model ultrasonically activated shears for hemostatic sectioning during video-assisted thoracic surgery. Gen Thorac Cardiovasc Surg. 2007;55(12):518-520.

8. Noble EJ, Smart NJ, Challand C, Sleigh K, Oriolowo A, Hosie KB. Experimental comparison of mesenteric vessel sealing and thermal damage between one bipolar and two ultrasonic shears devices. $\mathrm{Br} J$ Surg. 2011;98(6):797-800.

9. Sinha UK, Gallagher LA. Effects of steel scalpel, ultrasonic scalpel, $\mathrm{CO} 2$ laser, and monopolar and bipolar electrosurgery on wound healing in guinea pig oral mucosa. Laryngoscope. 2003;113(2):228-236.

10. Družijanić N, Pogorelić Z, Perko Z, Mrklić I, Tomić S. Comparison of lateral thermal damage of the human peritoneum using monopolar diathermy, Harmonic scalpel and LigaSure. Can J Surg. 2012;55(5): 317-321.

11. Rubino LJ, Konstantakos EK, Stills HF Jr, Dudley ES, Grunden BK, Malaviya P. Healing of iatrogenic skeletal muscle wounds is affected by incision device. Surg Innov. 2010;17(2):85-91.

12. Usas A, Usaite D, Gao X, Huard J, Clymer JW, Malaviya P. Use of an ultrasonic blade facilitates muscle repair after incision injury. $J$ Surg Res. 2011;167(2):e177-e184.

13. Vetere PF, Lazarou G, Mondesir C, Wei K, Khullar P, Ogden L. Strategies to minimize adhesion formation after surgery. JSLS. 2011;15(3):350-354.
Medical Devices: Evidence and Research

\section{Publish your work in this journal}

Medical Devices: Evidence and Research is an international, peerreviewed, open access journal that focuses on the evidence, technology, research, and expert opinion supporting the use and application of medical devices in the diagnosis, treatment and management of clinical conditions and physiological processes. The identification of novel

\section{Dovepress}

devices and optimal use of existing devices which will lead to improved clinical outcomes and more effective patient management and safety is a key feature. The manuscript management system is completely online and includes a quick and fair peer-review system. Visit http://www. dovepress.com/testimonials.php to read real quotes from authors. 